\title{
SCATTERING OF HIGH VELOCITY ELECTRONS BY THIN FOILS
}

\author{
By H. V. NeHER \\ Norman Bridge Laboratory, California Institute of Technology
}

(Received August 25, 1931)

\begin{abstract}
With a narrow, homogeneous beam of electrons, scattering by thin foils of aluminum, silver and gold has been investigated. Voltages up to $145 \mathrm{kv}(\beta=0.63)$ were used. Under conditions where single scattering was predominate and secondary electrons were absent, the amount of scattering was studied as a function of the primary energy, atomic number and angle. Absolute values were also obtained. The above investigations have lead to the following results: (1) Secondary electrons are defined and a means of eliminating them is proposed and used. (2) Wentzel's criterion for single scattering is tested over a wide range of energies. The value of $\theta / 4 \omega_{\min }$ for aluminum is found to increase from 3.3 at $45 \mathrm{kv}$ to 6.1 at $145 \mathrm{kv}$. (3) A more critical criterion for single scattering by thin foils is obtained which depends on the shape of the curve connecting $\rho$, the amount of scattering, with angle. (4) Dependence of scattering on energy of primary beam is found to agree well with either Mott's equation or with the relation $k / V^{2}$, but is at variance with the classical relativistic theory. (5) Comparison of values of scattering for aluminum, silver and gold shows that $\rho$ increases faster than $Z^{2}$. (6) Scattering is obtained as a function of angle from $95^{\circ}$ to $173^{\circ}$. For aluminum the dependence found experimentally agrees well with either Mott's or Rutherford's equation. The latter also gives the correct dependence on angle for silver and gold. Mott's equation is not applicable for these heavy elements. (7) Absolute values of scattering for aluminum compared with theory give $\rho=1.32$ of the value given by Mott's equation. This relation is valid within the ranges $\theta=95^{\circ}-173^{\circ}, V=56-145 \mathrm{kv}$. (8) Secondary electrons coming from the foil are distributed according to the simple cosine law. (9) No evidence of loss of energy due to radiation is found up to one-half the energy of the primary beam.
\end{abstract}

\section{INTRODUCTION}

$T$ $\mathrm{HE}$ experimental study of scattering of high velocity electrons by atomic nuclei is far more difficult than the corresponding problem using $\alpha$-particles. At least three fundamental complications are encountered when electrons are used for the bombarding particles. First, the relativity change of mass of the electron becomes increasingly important at energies above 40,000 volts. Second, secondary electrons emitted by the scatterer are indistinguishable from the primary electrons scattered by the nuclei. Third, effects due to loss of energy of the primary electron through radiation may be important for the light elements and large angles.

Investigations on scattering of high velocity electrons have been made with both $\beta$-rays and cathode rays. Chadwick and Mercier ${ }^{1}$ using radium $E$ as a source of $\beta$-rays obtained approximate agreement with the classical,

${ }^{1}$ Chadwick and Mercier, Phil. Mag. 50, 208 (1925). 
theoretical prediction that the amount of scattering should increase with the square of the atomic number. Comparison of absolute values with Darwin's classical relativistic equation showed the experimental results to be 25 percent too large. However, a lack of homogeniety of the $\beta$-rays together with the difficulties encountered from the $\gamma$-rays make scattering experiments with radioactive sources unreliable. Schonland, ${ }^{2}$ using cathode rays with energies up to $79 \mathrm{kv}$ obtained agreement with Darwin's theory for absolute magnitude of the scattering as well as a correct dependence upon energy and atomic number.

Because of the lack of sufficient and accurate data and the increased theoretical interest in the problem, the following more extended investigations were undertaken.

\section{Theories of Nuclear Scattering}

Rutherford's equation giving the probability of an $\alpha$-particle of mass $m$ and charge $2 e$ being scattered within the solid angle $d \omega$ at an angle $\theta$ to the original direction of motion by a nucleus of atomic number $Z$ may be written as follows:

$$
d \rho=\frac{n t Z^{2} e^{4}}{m^{2} v^{4}} \operatorname{cosec}^{4} \frac{\theta}{2} d \omega,
$$

where $n$ is the number of nuclei per $\mathrm{cm}^{3}, t$ is the thickness of matter traversed and $v$ is the velocity of the particle. This equation does not include a relativity correction and is not applicable in the case of high velocity electrons where $\beta$ is large.

Darwin $^{3}$ has calculated the orbit of a high velocity electron in the field of a heavy positive nucleus, taking into account change of mass as the electron passes the nucleus. He arrived at the result that if the electron comes within a certain critical distance $p_{0}$ it will spiral in and be absorbed by the nucleus. This can have no physical meaning since no transmutation of the elements is observed. Using Darwin's result, Crow ther and Schonland ${ }^{4}$ deduced the angular distribution of the electrons scattered by nuclei, neglecting those which spiraled into the nucleus. The value for the scattering between $90^{\circ}$ and $160^{\circ}$ for $\mathrm{Al}, \mathrm{Cu}$ and $\mathrm{Ag}$ found experimentally by Schonland ${ }^{5}$ agreed well with that deduced from Darwin's orbits. Later Schonland ${ }^{6}$ showed that these spiralling electrons could not be neglected in his case and in order to get a solution to the problem he assumed that they emerged uniformly in all directions. This gave a result at variance with his experimental work.

The equation of Crowther and Schonland, both in the original and in the latter form given by Schonland, gives a dependence on $\beta$ and $\theta$ as well as an absolute magnitude not found in this work. There is also some confusion as

2 B. F. J. Schonland, Proc. Roy. Soc. A113, 87 (1926-27).

${ }^{3}$ C. J. Darwin, Phil. Mag. 25, 201 (1913).

${ }^{4}$ J. A. Crowther and B. F. J. Schonland, Proc. Roy. Soc. A100, 526 (1921-22).

5 Reference 2.

${ }^{6}$ B. F. J. Schonland, Proc. Roy. Soc. A119, 673 (1928). 
to the form of the correction to be applied. ${ }^{7}$ In addition, from considerations of wave mechanics, it appears quite clear that we can no longer regard the electron as a point when the distances of approach become of the order of $h / m v$, the wave-length to be associated with the electron. Comparison of Darwin's relativistic scattering equation with experiment is given in Table IV. It will be noticed that the relative as well as the absolute magnitudes do not agree over a wide range of energies. Consequently we shall not consider this theory further.

Perhaps the best treatment of the problem has been given by Mott ${ }^{8}$ who uses Dirac's wave equation which includes corrections for both relativity and spin. The result arrived at may be expressed as

$$
\begin{aligned}
d \rho=\frac{n t Z^{2} e^{4}}{4 m_{0}{ }^{2} c^{4}}\left(\frac{1-\beta^{2}}{\beta^{4}}\right)\left[\operatorname{cosec}^{4}\right. & \frac{\theta}{2}-\beta^{2} \operatorname{cosec}^{2} \frac{\theta}{2} \\
& \left.+\beta \pi \frac{2 \pi e^{2}}{h c} Z \frac{\cos ^{2} \theta / 2}{\sin ^{2} \theta / 2}+\text { terms in }\left(\frac{2 \pi e^{2}}{h c} Z\right)^{2}\right] .
\end{aligned}
$$

For the angles $\theta_{1}$ and $\theta_{2}$ this becomes,

$$
\begin{aligned}
\dot{\rho}=\frac{\pi n t Z^{2} e^{4}}{m_{0}^{2} c^{4}}\left(\frac{1-\beta^{2}}{\beta^{4}}\right)\left[\cot ^{2} \frac{\theta_{1}}{2}-\cot ^{2} \frac{\theta_{2}}{2}-2 \beta^{2} \log \frac{\sin \theta_{2} / 2}{\sin \theta_{1} / 2}\right. \\
\left.+\frac{2 \pi \beta Z}{137}\left(\sin \frac{\theta_{1}}{2}+\operatorname{cosec} \frac{\theta_{1}}{2}-\sin \frac{\theta_{2}}{2}-\operatorname{cosec} \frac{\theta_{2}}{2}\right)+\cdots\right] .
\end{aligned}
$$

The quantity $\rho$ in this equation gives the ratio of the number of electrons scattered between the angles $\theta_{1}$ and $\theta_{2}$ to the total number of electrons incident on the scatterer.

\section{Description of Apparatus}

The apparatus in general is similar to that used by Schonland ${ }^{9}$ for studying the same problem. A general view of the apparatus is shown in Fig. 1.

\section{The electron "gun".}

All metal parts were turned from copper. This metal has the advantages of being nonmagnetic as well as having a low vapor pressure. To eliminate the possibility of cold emission all metal parts were given two coats of nickel, then plated with chronium and finally given a very high polish. Metal to glass seals were used throughout. This eliminated entirely waxes and greases and made possible the attaining of a high vacuum in a minimum time. The metal shield extending back over the cathode was designed to protect the glass. No difficulty was experienced when working at $145 \mathrm{kv}$ after the tube had been outgassed by continuous operation at lower voltages.

The filament was of the concentrated type wound with 10 mil tungsten

${ }^{7}$ E. E. Rutherford, Radiation from Radioactive Sources, 225 (1930).

${ }^{8}$ N. F. Mott, Proc. Roy. Soc. A124, 425 (1929).

${ }^{9}$ See reference 2 and B. F. J. Schonland, Proc. Roy. Soc. 108, 187 (1925). 
wire in the form of a helix and bent back on itself. This type was found to give a more intense beam finally emerging in to the scattering chambers than that given by a number of other designs constructed. The position of the filament in relation to the end of the metal tube in which it was situated had to be adjusted very accurately to secure a maximum of current. It was also found that

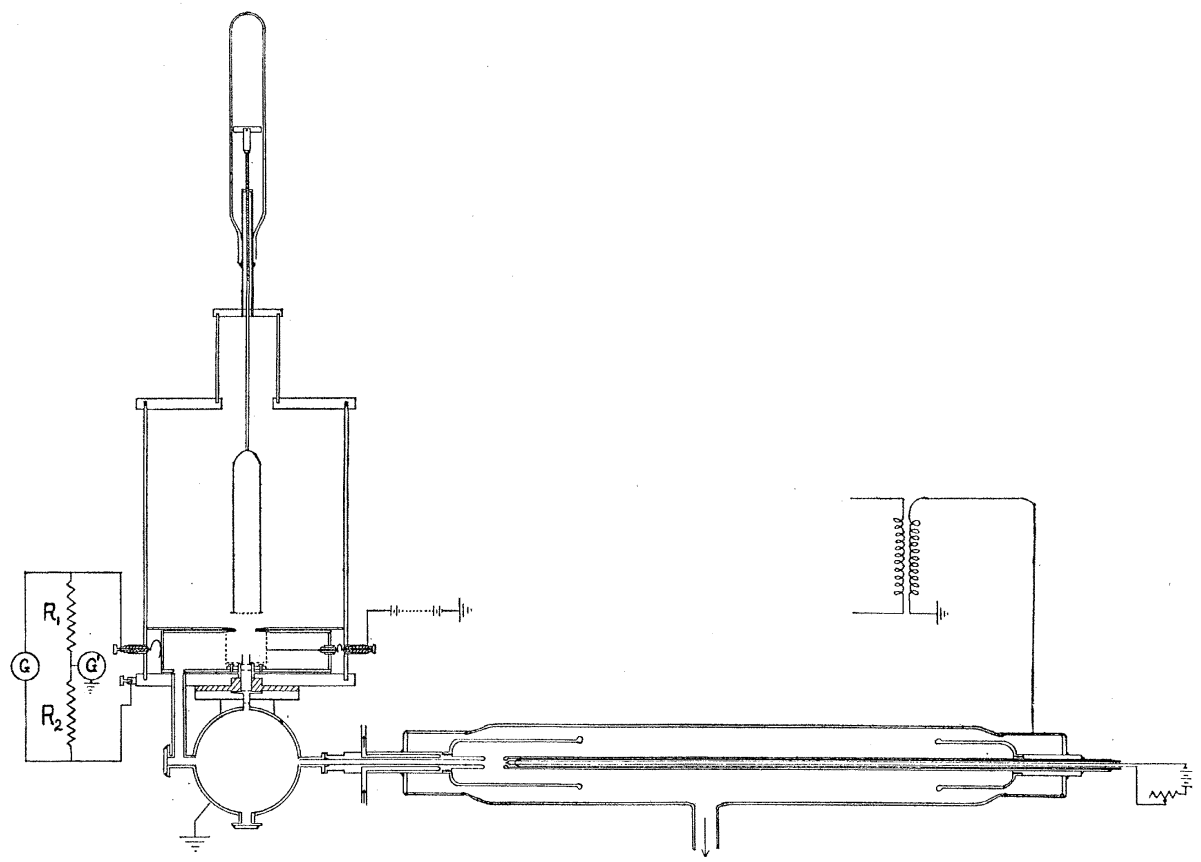

Fig. 1. Cross-section of apparatus showing electron-gun, solenoid, scattering chambers, method of varying angle and electrical connections.

a resistance of several hundred thousand ohms, placed between the filament and the metal tube surrounding it, increased the focusing action of the electric field between the cathode and anode and very materially strengthened the current received into the scattering chambers for a given total emission.

\section{The solenoid.}

This consisted of a brass cylinder $13 \mathrm{~cm}$ in diameter and $50 \mathrm{~cm}$ long wound with two layers of copper wire $1.63 \mathrm{~mm}$ in diameter. A section $10 \mathrm{~cm}$ long in the middle of the cylinder was partitioned from the remaining and could be evacuated with the rest of the apparatus. Each end of the solenoid was water cooled. Special precautions were taken to eliminate all magnetic substances in the neighborhood of the solenoid, since the maximum field was only 250 gauss.

As the solenoid was used not only as a means of obtaining a homogeneous beam but also for measuring the voltage of the electrons, it was necessary to know its constant. For an electron bent in a magnetic field,

$$
(H \rho)=\frac{m_{0} v}{e\left(1-\beta^{2}\right)^{1 / 2}},
$$


while the energy of the electron after falling through a potential $V$ is,

$$
V e=m_{0} c^{2}\left(\frac{1}{\left(1-\beta^{2}\right)^{1 / 2}}-1\right) .
$$

Eliminating $\beta$ between these two equations,

$$
(H \rho)^{2}=\frac{1}{c^{2}} \cdot \frac{2 m_{0} c^{2}}{e}\left(V+\frac{e}{2 m_{0} c^{2}} V^{2}\right) .
$$

At low voltages,

$$
(H \rho)^{2}=k I^{2}=k^{\prime} V
$$

The relation between high and low voltages can then be written in terms of the respective currents in the solenoid as,

$$
I^{2}=\frac{1}{K}\left(V+0.982 \times 10^{-6} V^{2}\right)
$$

where $K=V / I^{2}$ for low voltages. $V$ has been expressed in volts, $I$ in amperes and the values of the constants inserted. The quantity $K$ will be defined as the constant of the solenoid. Calibrations were made at high voltages with a standard sphere gap and by an electrical deflection method. The two methods gave results agreeing to within $1 / 2$ percent. The constant obtained was,

$$
K=737 \pm 3 \text { volt amp. } .^{-2} \text {. }
$$

\section{Scattering chambers.}

The arrangement of the scattering chambers can best be described by referring to Fig. 2. The purpose of using chambers of such large diameter

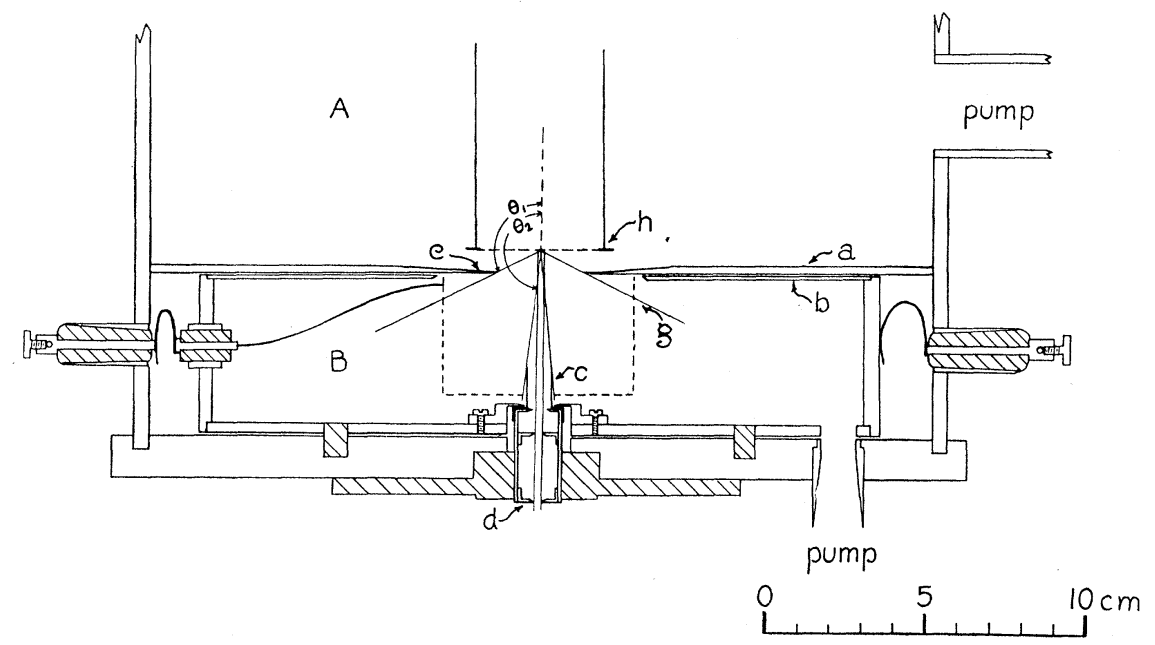

Fig. 2. Enlarged view of lower scattering chamber showing the relations of scatterer, grid and collector. The angles between which the scattered electrons were collected are also shown.

(20 and $25 \mathrm{~cm}$ ) was to eliminate as far as practical the effect of reflected electrons going from one into the other. When the inside was brass the reflection 
from the upper into the lower chamber amounted to $0.0003-0.0009$ of the main beam. When the whole inside was lined with aluminum this value was reduced to $0.0001-0.0003$.

The electrons were admitted from the solenoid into the scattering chambers through four collimating openings made of aluminum which were all grounded. The first three openings were $2.8 \mathrm{~mm}$ in diameter and the last was $4 \mathrm{~mm}$. The purpose of this last opening was to stop scattered electrons from the openings below entering the lower chamber. The small, thin aluminum cylinder $c$ was fastened to $d$ and extended up into $B$ a distance of $1.8 \mathrm{~cm}$. It performed two functions: (1) it further prevented stray electrons from the openings below reaching $B$, and (2) it definitely fixed the larger angle of scattering.

Chamber $B$ was insulated from $A$ by thin $(0.05 \mathrm{~mm})$ mica disks. The metal disks $a$ and $b$ were fastened to $A$ and $B$ respectively. A thin aluminum ring $0.015 \mathrm{~cm}$ thick, $0.8 \mathrm{~cm}$ wide, with a $2.83 \mathrm{~cm}$ opening in its center formed the final separation between $A$ and $B$. The disk $b$ had an opening in it slightly larger than the grid $g$. This was found necessary since many electrons collect in the space within the grid and part of them would be collected by $B$ if $b$ extended beyond the edge of $g$.

\section{Grids to stop secondary electrons.}

For investigating secondary electrons up to 2000 volts, a wire grid was constructed. It consisted of a $4 \mathrm{~mm}$ mesh cylindrical framework made of 2

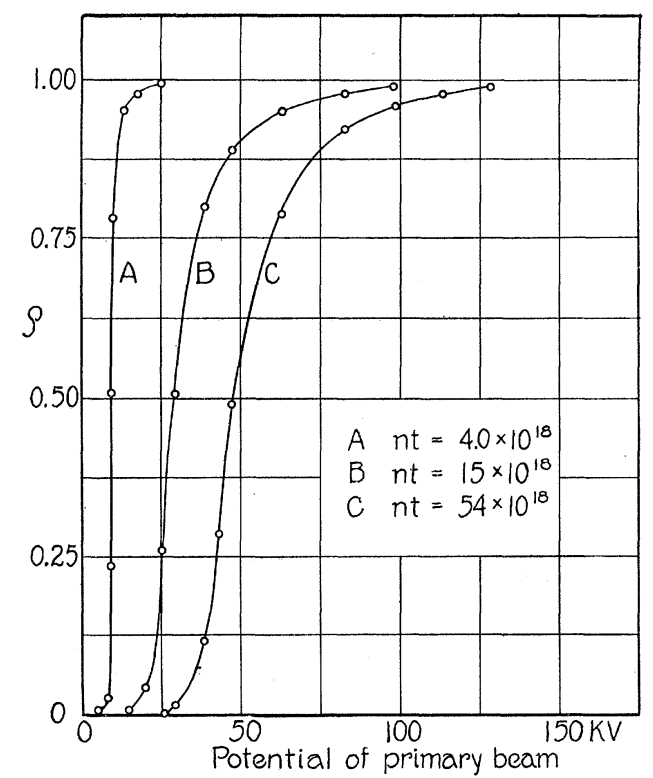

Fig. 3. The stopping power of aluminum foils of different thicknesses. The "range" may be obtained approximately from the lower knee of the curve.

mil nickel wire. The ratio of wire to total space was 0.025 . The reflection coefficient of nickel is 0.30 , hence a correction of 1.6 percent was applied to the readings when this grid was used. 
When secondary electrons with energies greater than 2000 volts were investigated, because of the ionization currents produced and the attendant insulation difficulties, other means were resorted to than that of using actual potentials. Such a means was furnished by thin foils. Referring to Fig. 3 it will be noticed from the curve for aluminum connecting $\rho$ (the ratio of transmitted electrons to the total number incident) with the voltage of the primary beam, that no electrons are transmitted up to a certain voltage after which there is a sudden increase. At voltages ordinarily used, from 90 to $145 \mathrm{kv}$, even a piece of aluminum $0.01 \mathrm{~mm}$ thick is very transparent and at the same time acts as an equivalent stopping potential of 50 to $55 \mathrm{kv}$. A small correction for voltages below $120 \mathrm{kv}$ can be applied to account for those electrons scattered elastically which are stopped by this foil if it is used as a grid to stop secondary electrons. Although such foils do not form a barrier as perfect as would be furnished by an actual stopping potential, they do afford a means

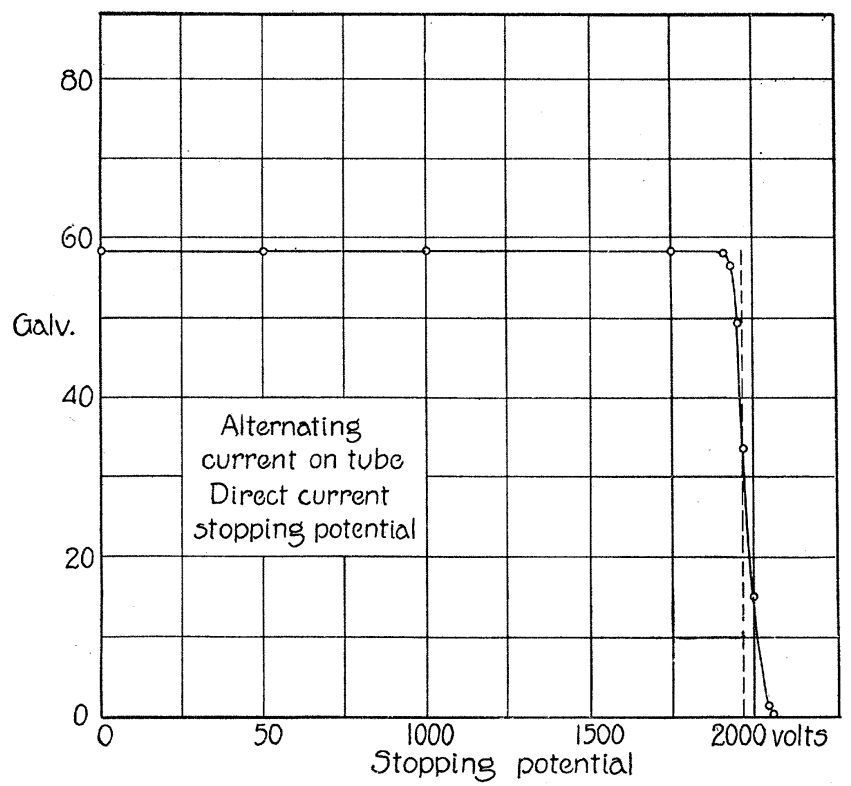

Fig. 4. Homogeniety of electron beam shown by applying D. C. stopping potentials. Dotted line represents computed voltage from known constant of solenoid.

of obtaining equivalent stopping potentials with an accuracy sufficient for the problem under consideration. This will appear more clearly in what follows.

Secondary electrons will also be set free from the foil grid on the collector side by the electrons which go through. To investigate this point a fine wire grid was placed around the foil grid and a stopping potential of 2000 volts applied. A decrease in the current received in $B$ of 1 to 2 percent was found if the primary voltage was of the order of $50 \mathrm{kv}$, but for $100 \mathrm{kv}$ the effect decreased to 0.3 to 0.4 percent. When necessary this correction was applied to the readings.

A means is thus provided by which secondary electron velocities up to 
one-half the energy of the initial beam can be investigated. Reasons will be given later why one-half the energy of the initial beam is the upper limit to the energies of the secondary electrons.

\section{Method of varying angle.}

The foil acting as the scatterer was mounted on a thin metal ring, $h, 3 \mathrm{~cm}$ in diameter, supported by a nickel wire $0.6 \mathrm{~mm}$ in diameter. The whole could be raised and lowered by means of a magnetic control as shown in Fig. 1, The height of the foil above the opening $e$ could be measured to within $0.008 \mathrm{~cm}$. The screw on the control mechanism was calibrated with a traveling microscope. The angles between which the electrons were collected were determined by the size of the openings $c$ and $e$ and by the height of the foil.

\section{Homogeniety of beam.}

The homogeniety of the beam for the case of alternating current applied to the tube was tested with d.c. stopping potentials. The result is shown in Fig. 4. Such a test was desirable since an alternating current transformer was used as a source of high voltage. The dotted line in the figure represents the voltage of the electrons computed from the constant of the solenoid. It will be noticed that the distribution of energies is almost symmetrical about the computed value. If $\pm \Delta V$ represents the heterogeneity of the beam, then if each side of the true value is considered, (cf. Eq. (1))

$$
\begin{aligned}
\rho^{\prime} & =\frac{k}{(V+\Delta V)^{2}}=\frac{k}{V^{2}}\left(1-\frac{2 \Delta V}{V}+\cdots\right) \\
\rho^{\prime \prime} & =\frac{k}{(V-\Delta V)^{2}}=\frac{k}{V^{2}}\left(1+\frac{2 V}{V}+\cdots\right) \\
\frac{\rho^{\prime}+\rho^{\prime \prime}}{2} & \doteqdot \frac{k}{V^{2}}=\rho .
\end{aligned}
$$

If the beam of electrons is symmetrical about a mean voltage given by the constant of the solenoid, this same reasoning will apply to all $\pm \Delta V$ 's and if the spread of voltage is not more than 5 percent on either side, the error introduced into the experimental results due to the lack of homogeniety will be negligible.

\section{Determination of nt.}

It was soon found that the variations in the thickness of the foils were too great to permit weighing a large sheet of the material and computing the average value of $n t$. Consequently a quartz torsion balance which had a constant of $0.1214 \times 10^{-6} \mathrm{gm} / \mathrm{div}$. was constructed. The constant was determined by weighing small sections of very fine wire a long piece of which had been previously weighed on an analytical balance. Aluminum foils $5 \times 10^{-5}$ $\mathrm{cm}$ thick and $3 \mathrm{~mm}$ square could be weighed to 1 percent.

If $m$ is the mass of a piece of foil of area $A$, then the product $n t$ is given by: $n t=6.06 \times 10^{23} \mathrm{~m} / M A$, where $M$ is the atomic weight of the element compos- 
ing the foil. The value of $n t$ which enters directly in to the scattering equation, is independent of the density of the material. Since that portion of the foil where the beam passed through was cut out and weighed, local variations in uniformity should not introduce a large error if the beam were uniform. This point was tested with the thinnest foils by rotating the foil one turn and taking readings every $45^{\circ}$. Variations of not more than 2 percent were observed while the average variation was 1 percent.

\section{Experimental Procedure}

The current in the solenoid was set and kept at a constant value while the voltage of the electron beam was increased until a maximum current came through into the scattering chambers as indicated by the galvanometer $G^{\prime}$. (See Fig. 1), $R_{2}$ was kept constant at $10^{4} \mathrm{ohms}$ and $R_{1}$ adjusted until $G$ read zero deflection. A change of resistance in $R_{1}$ of $0.1 \mathrm{ohm}$ would give a deflection of $G$ of approximately $5 \mathrm{~mm}$. Since $R_{2}$ was $10^{4} \mathrm{ohms}$, changes in $\rho$ of 1 part in $10^{6}$ of the main beam could be detected. When a balance of $G$ was obtained, the drop in potential across $R_{2}$ was the same as that across $R_{1}$ and the apparent value of scattering within the given angles was then given by,

$$
\rho=\frac{R_{1}}{R_{1}+R_{2}} .
$$

To this value of $\rho$ several corrections must be applied.

1. A correction for the value of $\rho$ when no foil was present. It varied with the height of the ring $h$, the kind of foil used as a stopping potential and the potential of the primary beam. This "zero correction" was checked at various times. A typical selection of values is given in the table below for a foil with an equivalent stopping potential of $-27,000$ volts.

TABLE I.

\begin{tabular}{c|c|c|c}
\hline$V$ & $\theta_{1}$ & $\theta_{2}$ & $\rho$ (correction) \\
\hline $68.9 \mathrm{kv}$ & $95^{\circ} 10^{\prime}$ & $172^{\circ} 5^{\prime}$ & 0.00022 \\
96.9 & $95^{\circ} 10^{\prime}$ & $172^{\circ} 5^{\prime}$ & 0.00026 \\
129.0 & $95^{\circ} 10^{\prime}$ & $172^{\circ} 5^{\prime}$ & 0.00028 \\
145.0 & $95^{\circ} 10^{\prime}$ & $172^{\circ} 5^{\prime}$ & 0.00030 \\
\hline
\end{tabular}

This "zero correction" is to be subtracted from the apparent value of $\rho$.

2. A correction for the wire of which the grid was constructed. It amounted to 0.8 percent for the foil covered grids and 1.6 percent for the fine mesh wire grid. This correction is to be added.

3. A correction for the stopping power of the foil grids for elastically scattered electrons. This was obtained from Fig. 3 and amounted at most to only a few percent. This is to be added.

4. A correction for the reflection out of chamber $B$ and for the absorption by the foil grid of electrons reflected from the walls of $B$. Two experimental methods could be used to determine the magnitude of these two effects. (1) The dimensions of the scattering chamber could be increased and an extrapolation to an infinitely large one made; or, (2) the chamber could be lined 
with different metals of known reflection coefficients and an extrapolation to zero reflection made. The latter method was chosen as the more practical. Consequently, the reflection coefficients of aluminum and brass were determined as well as the angular distribution of these reflected electrons. The ratio of the coefficients for aluminum and brass is $0.13 / 0.29=0.45$. A typical example showing the effect of lining the chamber $B$ with brass and aluminum is given below:

\begin{tabular}{l|c|c|c|c|c}
\hline \hline Metal & $V$ & $\theta_{1}$ & $\theta_{2}$ & $V_{s}$ & $\rho$ \\
\hline Al & $96.9 \mathrm{kv}$ & $108^{\circ}$ & $173^{\circ}$ & $\begin{array}{l}-27 \mathrm{kv} \\
-27 \mathrm{kv}\end{array}$ & $\begin{array}{l}0.001260 \\
0.001253\end{array}$ \\
\hline
\end{tabular}

The effects increased slightly for lower voltages and decreased for higher voltages. In most cases the correction was negligible.

When the foil grid was used it was connected electrically to chamber $A$. This was necessary since $B$ was to collect only those electrons scattered elastically while $A$ was to collect all others. Secondaries emitted from the foil or other parts of $A$ subtracted in one place but added in another so that the net result was nil.

\section{Secondary electrons.}

\section{RESULTS}

It is of great importance to eliminate the effect of secondary electrons if nuclear scattering is to be studied. When stopping potentials are applied to the electrons coming from a thin metal foil which is being bombarded with a homogeneous beam of high velocity electrons, it is found that the electrons so emitted have a wide distribution of energies. A large number have energies below 100 volts, but an appreciable number, compared with the electrons which have been scattered elastically, have much higher energies. It is necessary to determine how high a stopping potential must be applied to stop all secondary electrons and still not stop those scattered elastically.

Secondary electrons have been defined by other investigators in various ways. Becher ${ }^{10}$ and Stehberger, ${ }^{11}$ working at energies below 12,000 volts defined as secondary electrons all those with energies below 36 volts. Wagner ${ }^{12}$ defined all electrons coming from the material bombarded as secondary. In this paper a different definition based upon the process of collision of two electrons will be adopted. It is well known that as a purely mechanical process, when two electrons collide, one of them being initially at rest, they part at an angle of $90^{\circ}$ to one another. We shall distinguish them after collision by defining the one with the greater energy as the primary and the one with the lesser energy as the secondary.

It will be apparent from the above considerations that for a sufficiently thin foil, many secondary electrons formed in the center of the foil will have

${ }_{10}$ A. Becher, Ann. d. Physik 78, 288 (1925).

${ }^{11}$ K. H. Stehberger, Ann. d. Physik 86, 825 (1928).

12 P. B. Wagner, Phys. Rev. 35, 98 (1930). 
sufficient energy to emerge. If stopping potentials equal to one-half the energy of the initial beam are applied it is certain that all secondaries are stopped and that the remaining electrons are those which have been scattered by nuclei alone.

The effect of applying successively higher stopping potentials to the electrons emitted by thin foils of aluminum bombarded with $128 \mathrm{kv}$ electrons is shown in Fig. 5. The ordinate gives the ratio of the number of electrons penetrating the stopping potential (given by the corresponding abscissa) to the total number of primary electrons incident on the scatterer. The angles between which the scattered particles were collected in each case were $95^{\circ}$ and $172^{\circ}$. The values of $\rho$ given in Fig. 5 refer only to curve $(a)$.

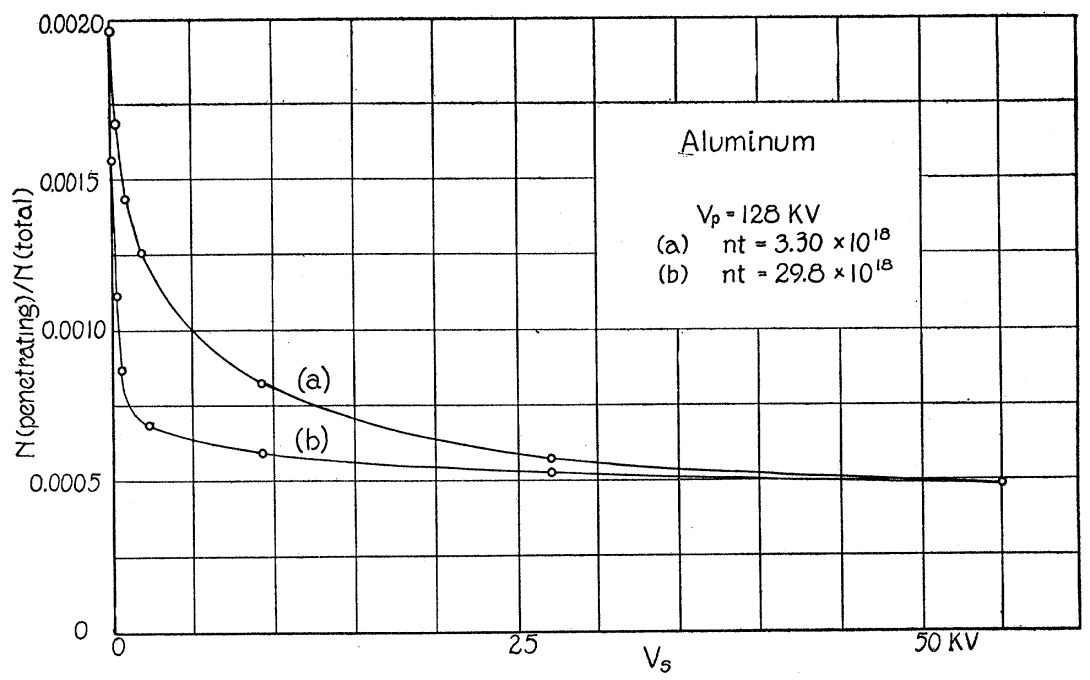

Fig. 5. Shows (1) that an appreciable number of secondary electrons are present from a thin foil at very high energies, (2) that secondary electrons are more important for a thinner foil. Curve (b) fitted to $(a)$ at $55 \mathrm{kv}$.

The relative importance of secondary electrons for thin foils is greater than for thicker foils. This is shown in curve $(b)$ where the foil was eight times the thickness of that used in obtaining curve $(a) .(b)$ is fitted to $(a)$ at $55 \mathrm{kv}$. This difference of relative importance of secondary electrons may be explained by the greater probability of a secondary electron emerging from the thinner foil. In each case the probability of the nuclearly scattered primary electron emerging is practically 1.

It might be thought that the electrons stopped between 25 and $55 \mathrm{kv}$ were those which had been scattered by the nuclei but which had gradually lost sufficient energy to other electrons in the foil so that they were stopped by a potential less than half their original value. If this were so one would expect the curve for the thicker foil to nearly coincide with the one for the thinner foil. It therefore appears that there are a few secondary electrons at very high energies, although they form an appreciable percentage of the total number collected. 
It is interesting to note that Schonland used 150 volts stopping potential and assumed that all secondary electrons were eliminated.

\section{Single scattering.}

Experimentally if $\rho$ increases linearly with $n t$ single scattering is the predominating factor. The results of these tests are shown in Fig. 6. Wentzel ${ }^{13}$ has

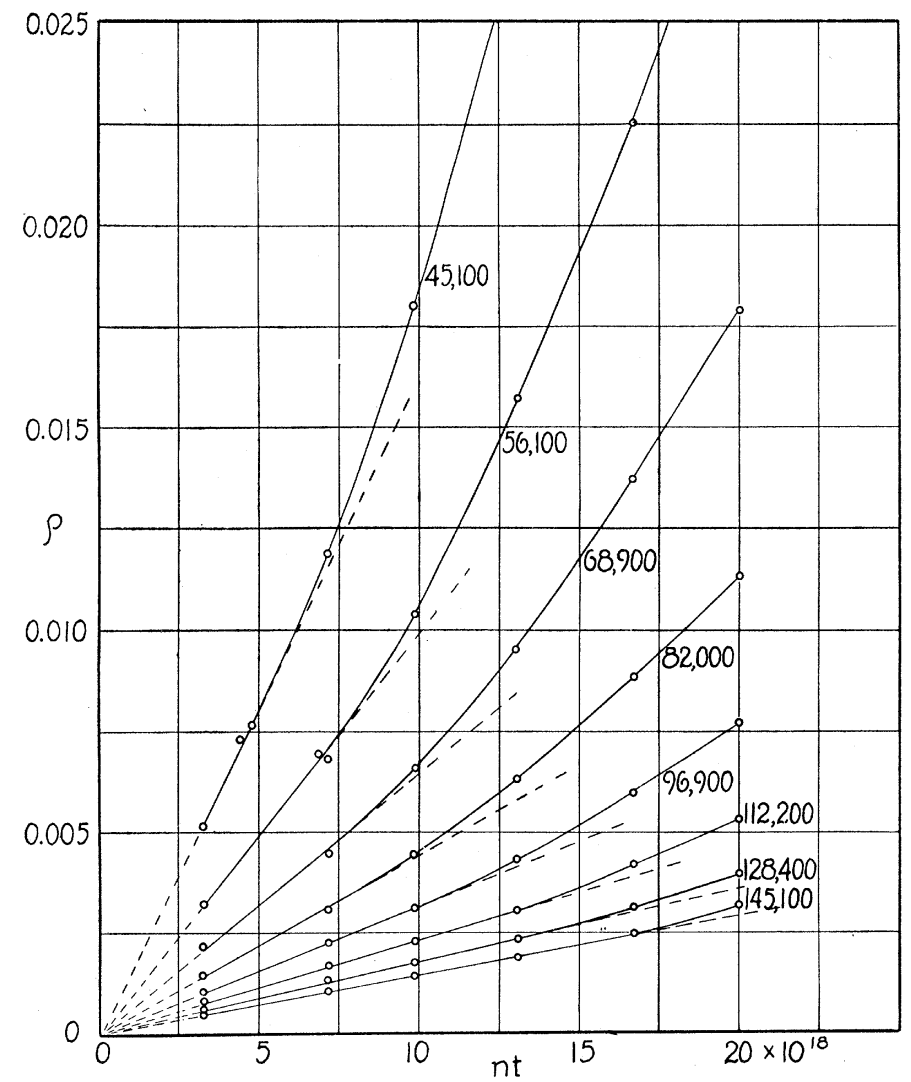

Fig. 6. Tests of single scattering- $\rho a n t$. Angles: $\theta_{1}=95^{\circ} 10^{\prime}, \theta_{2}=172^{\circ} 5^{\prime}$.

obtained a criterion based on classical reasoning which must be satisfied for single scattering to be the predominating factor. It may be stated as follows: If

$$
4 \omega_{\min }=8 \cot ^{-1} \frac{2 V}{Z e}\left(\frac{2}{\pi n t}\right)^{1 / 2},
$$

then for single scattering,

$$
\frac{\theta}{4 \omega_{\min }}=3 \text { or } 4
$$

If we apply Wentzel's criterion to the point where the curves depart from linearity in Fig. 10 we obtain the values given in Table II.

${ }^{13}$ G. Wentzel, Ann. d. Physik 69, 333 (1922). 

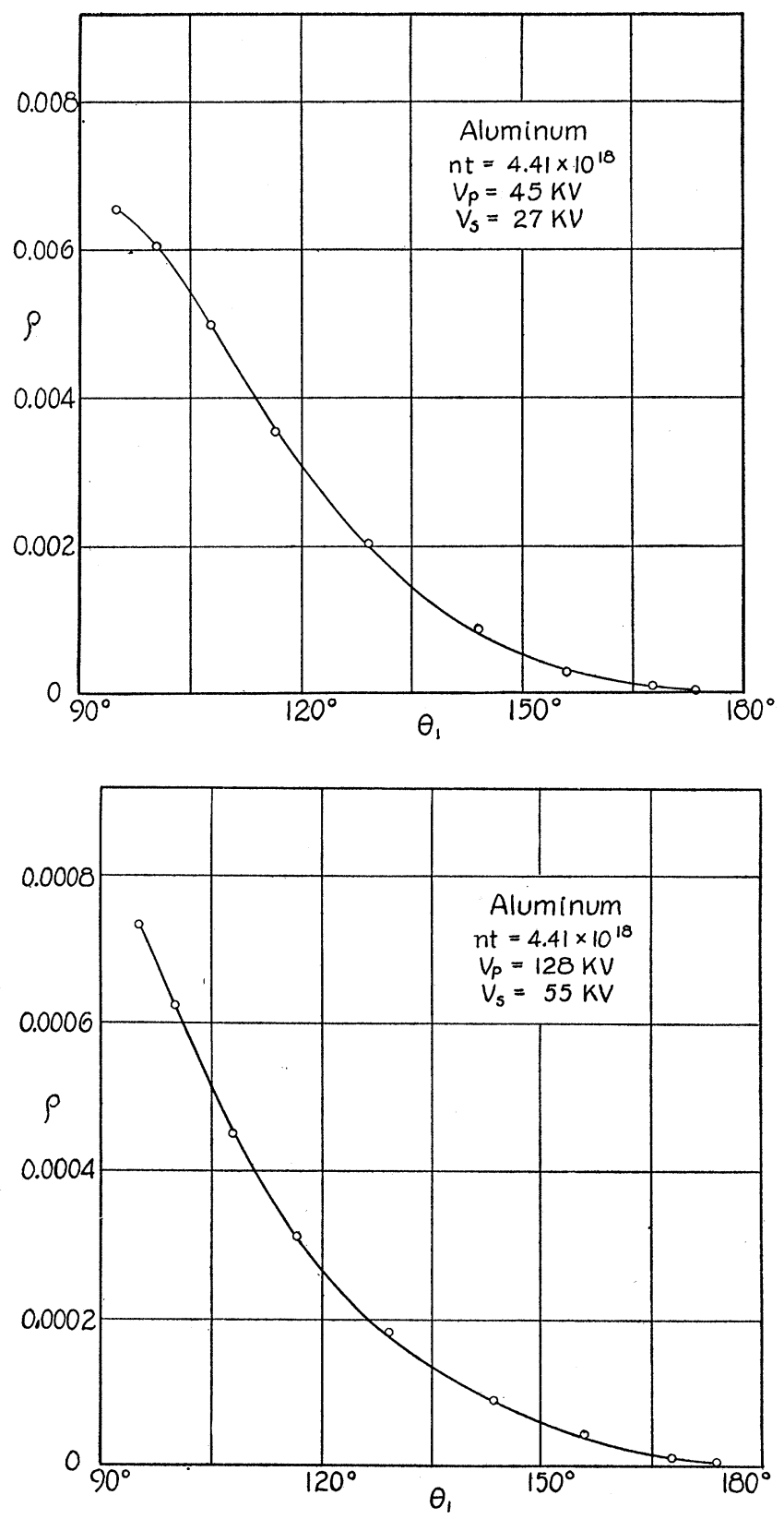

Figs. 7 and 8. More accurate test of single scattering by thin foils. Hump in Fig. 7 disappears for $V_{p}>90 \mathrm{kv}$ for a foil of this thickness as shown in Fig. 8. 
TABLE II. Aluminum.

\begin{tabular}{l|c|c|c}
\hline & $\theta_{1}=95^{\circ} 10^{\prime}$ & $\theta_{2}=172^{\circ} 5^{\prime}$ \\
\hline$n t$ & $V$ & $4 \omega_{\min }$ & $\theta_{1} / 4 \omega_{\min }$ \\
\hline $6.0 \times 10^{18}$ & 45.0 & $28^{\circ} 56^{\prime}$ & 3.3 \\
7.4 & 56.0 & $25^{\circ} 52^{\prime}$ & 3.7 \\
8.3 & 68.9 & $22^{\circ} 00^{\prime}$ & 4.2 \\
9.4 & 82.0 & $20^{\circ} 0^{\prime}$ & 4.7 \\
11.3 & 96.9 & $18^{\circ} 40^{\prime}$ & 5.0 \\
13.2 & 112.5 & $17^{\circ} 20^{\prime}$ & 5.5 \\
15.5 & 129.0 & $16^{\circ} 24^{\prime}$ & 5.8 \\
17.6 & 145.0 & $15^{\circ} 36^{\prime}$ & 6.1 \\
\hline \hline
\end{tabular}

The factor $\theta / 4 \omega_{\min }$ is not a constant but increases with the voltage. The value reported by Schonland is $\theta / 4 \omega_{\min }=3.0$, which is for both 60 and $80 \mathrm{kv}$. Judging from the results given here this value is much too low.

A more accurate criterion for single scattering from a foil can be obtained experimentally from the shape of the curve showing the variation of $\rho$ with angle. Near $90^{\circ}$ a scattered electron emerging from the foil must go a greater distance through the metal than one coming out at larger angles, and the probability of a second collision is increased. This will have the effect of decreasing the slope of the curve near $90^{\circ}$. These plurally scattered electrons will be partially thrown into larger angles and will give the hump shown in Fig. 7, which is for 45,000 volts with a stopping potential of 27,000 . Fig. 8, for the same foil $\left(n t=4.41 \times 10^{18}\right)$ but a primary voltage of 128,000 and a stopping potential of 55,000 , shows how the hump has completely disappeared. The shape of the curve for this particular thickness of aluminum foil remains unchanged from 90 to $145 \mathrm{kv}$. A necessary and sufficient condition for single scattering at angles from $90^{\circ}$ to $180^{\circ}$ when a foil is bombarded normally is that the curve connecting $\rho$ with $\theta$ shall not change its shape when the primary potential is increased.

TABLE III. Aluminum.

\begin{tabular}{l|c|c|c|c|c|c|c}
\hline \multicolumn{8}{c}{$V_{s}=-27,000 \mathrm{kv}, \theta_{1}=95^{\circ} 10^{\prime}, \theta_{2}=172^{\circ} 5^{\prime}$} \\
\hline $\mathrm{kv}$ & 56.1 & 68.9 & 82.0 & 96.9 & 112.2 & 128.4 & 145.1 \\
\hline & 9.47 & 6.35 & 4.38 & 3.05 & 2.34 & 1.63 & $1.35 \times 10^{-22}$ \\
$\frac{\rho}{n t}$ & 9.65 & 6.41 & 4.31 & 3.14 & 2.29 & & \\
& 9.69 & 6.38 & 4.43 & 3.09 & 2.31 & 1.71 & 1.41 \\
& 8.70 & 6.31 & 4.17 & 2.97 & 2.31 & 1.72 & 1.35 \\
\hline \multirow{2}{*}{ av. } & 8.92 & 6.12 & 4.38 & 3.10 & 2.28 & 1.73 & 1.38 \\
\hline \hline
\end{tabular}

An indication of the accuracy obtainable, using foils of such thickness in each case that single scattering was predominate, is shown in the following table for five different foils. The value $\rho / n t$ should be constant for constant voltages, angles and atomic number. The stopping potential, $V_{s}$, in each case was 27,000 volts. 


\section{Dependence of scattering on energy of primary beam.}

The variation of the amount of scattering for aluminum between the angles of $95^{\circ} 10^{\prime}$ and $172^{\circ} 5^{\prime}$ as a function of the energy of the beam is shown in Fig. 9. Plotted in the same figure are the relations $k / V^{2}$ and the relative values computed from Mott's equation, each being fitted to the experimental value at $56 \mathrm{kv}$.

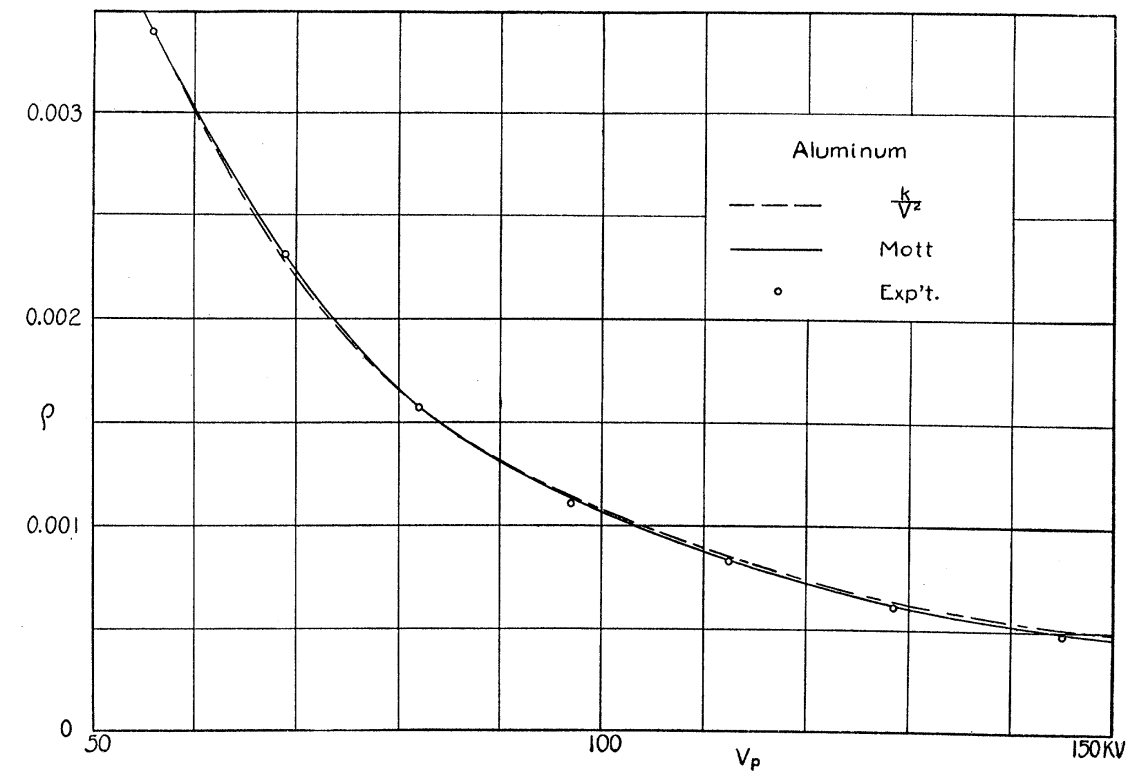

Fig. 9. Dependence of $\rho$ upon energy of primary beam with appropriate stopping potentials in each case.

TABLE IV. Aluminum.

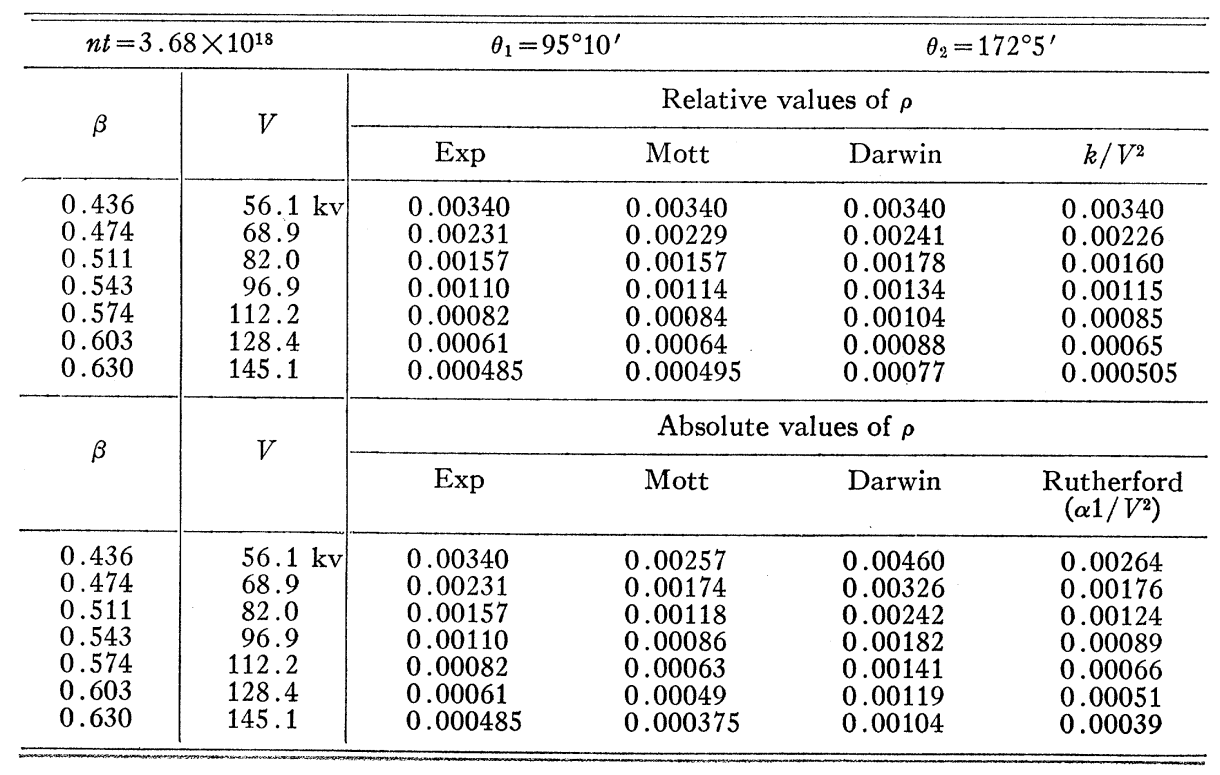


A comparison of the relative as well as the absolute values of $\rho$ is given in Table IV. The following points should be noted: (1) The dependence on energy of beam given by Mott's equation and by the empirical relation $k / V^{2}$ agrees well with experiment, while the equation based on relativity correction of the classical theory does not agree. (2) Absolute values of scattering within the angles given do not agree with any of the theories. Rutherford's equation cannot be written proportional to $1 / V^{2}$ above 30 to $40 \mathrm{kv}$. Mott's result is the one we shall consider most seriously. Comparing with the experimental values we obtain,

$$
\operatorname{Exp}=1.32 \text { Mott }
$$

which represents the facts quite closely for aluminum. It will be shown later that Mott's equation also gives the correct dependence on $\theta$. The above relation, then, is valid in the case of aluminum within the ranges, $V=56,000$ to 145,000 volts and $\theta=95^{\circ}$ to $173^{\circ}$.

\section{Dependence of scattering on $Z$.}

In Table $\mathrm{V}$ the factor $\rho / n t Z^{2} f(\theta / 2)$ is compared for $\mathrm{Al}, \mathrm{Ag}$ and $\mathrm{Au}$ at $\theta_{1}$ $=95^{\circ} 10^{\prime}$ and $\theta_{2}=172^{\circ} 5^{\prime}$ for $\beta=0.603$ and $\beta=0.630$. All the theories require that this factor should be a constant for all elements but it actually increases. Schonland also reports a value too high for gold and attributes it to an abnormal emission of secondary electrons. This explanation is hardly tenable for the results reported here because of the stopping potentials used. It may be pointed out here that Mott's equation applies best to the lighter elements and neglecting further terms in the expansion (see Eq. (2)) is scarcely justifiable in the cases of silver and gold.

TABLE $\mathrm{V}$.

\begin{tabular}{l|c|c|c|c|c|c}
\hline \hline & \multicolumn{3}{|c|}{$\beta=0.603$} & \multicolumn{3}{|c}{$\beta=0.630$} \\
\hline & $\mathrm{Al}$ & $\mathrm{Ag}$ & $\mathrm{Au}$ & $\mathrm{Al}$ & $\mathrm{Ag}$ & $\mathrm{Au}$ \\
\hline Mott & 1.52 & $\begin{array}{l}1.77 \\
1.65\end{array}$ & $\begin{array}{l}2.41 \times 10^{-24} \\
2.50\end{array}$ & $\begin{array}{c}1.24 \\
0.94\end{array}$ & $\begin{array}{c}1.38 \\
1.19\end{array}$ & $\begin{array}{c}1.95 \times 10^{-24} \\
\text { Ruth. }\end{array}$ \\
\hline \hline
\end{tabular}

In view of the fact that plural scattering contributed something to the value of $\rho$ for the thinnest silver and gold foils (800A and 1200A respectively) it would be expected that the value would be high. Wentzel's criterion for both these elements gives $4 \omega_{\min }=5.3$ at 128,000 volts. We should then expect mostly single scattering and the large values obtained for these elements must indicate that $\rho$ increases faster than $Z^{2}$.

\section{Dependence of scattering on angle.}

C. E. Eddy ${ }^{14}$ has studied the angular distribution of $\beta$-rays scattered by thin foils from $0^{\circ}$ to $50^{\circ}$ but under conditions where plural scattering was very prominent. Kemplerer ${ }^{15}$ working with voltages between $10 \mathrm{kv}$ and $40 \mathrm{kv}$

${ }^{14}$ C. E. Eddy, Proc. Camb. Phil. Soc. 25, 50 (1929).

15 Klemplerer, Ann. d. Physik 3, 849 (1929). 
found an angular dependence between $10^{\circ}$ and $120^{\circ}$ not given by any existing theory. The accuracy of this latter work, however, was probably insufficient to warrant conclusions. Certain definite angles have been used by other observers, but a consistent effort to obtain an accurate dependence on angle has not been made.

The dependence of scattering on angle found experimentally is well illustrated in Fig. 10 which is for aluminum. $\theta_{1}$ is plotted as abscissa so that any ordinate gives the value of the ratio of the number of electrons collected between $\theta_{1}$ and $\theta_{2}$ to the total number of electrons incident on the foil. $\theta_{2}$

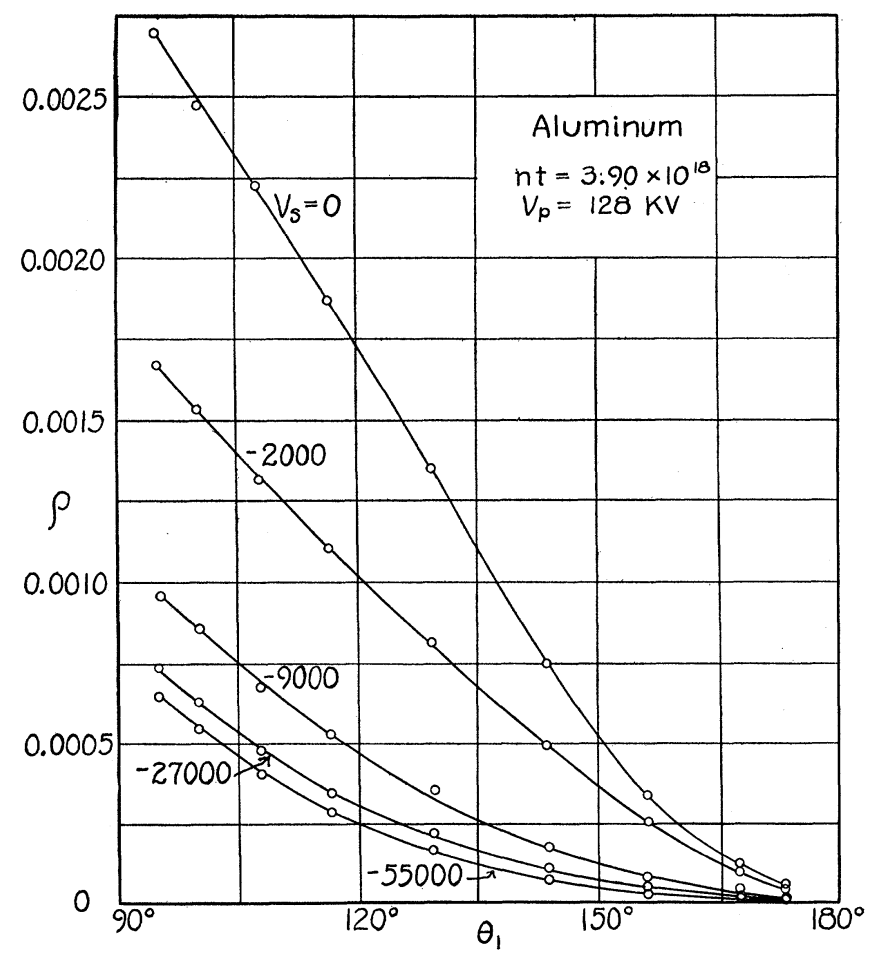

Fig. 10. Dependence of $\rho$ upon angle. Shows effect of applying successively higher stopping potentials both on absolute magnitude and shape of curve.

varies from $172^{\circ}$ when $\theta_{1}=95^{\circ}$, to $178^{\circ}$ when $\theta_{1}=173^{\circ}$. The primary voltage for each curve is 128,000 . The four curves plotted are for the stopping potentials given. In Fig. 11 plotted with values for $V_{s}=-55,000$ volts and fitted at $95^{\circ} 10^{\prime}$ is given the variation with $\theta$ from both Mott's and Rutherford's equations. All curves which have been obtained from 97 to $145 \mathrm{kv}$ are very similar in shape to the one given in Fig. 11 if the proper stopping potential is applied. At voltages below $97 \mathrm{kv}$, the hump shown in Fig. 7 begins to appear for the thinnest foil available, and no comparison with theory based on the assumption of single scattering can be made. For sufficiently high voltages either the equation of Mott or Rutherford gives a dependence on $\theta$ between $95^{\circ}$ and $173^{\circ}$ which agrees well with experiment. 
Similar curves have been obtained for gold and silver. The angular dependence for these two metals agrees well with Rutherford's $\cot ^{2} \theta / 2$ relation but the agreement is not as good with Mott's equation. This deviation is probably due to the fact that, as mentioned before, neglecting further terms in the expansion is not permissible for the heavy elements.

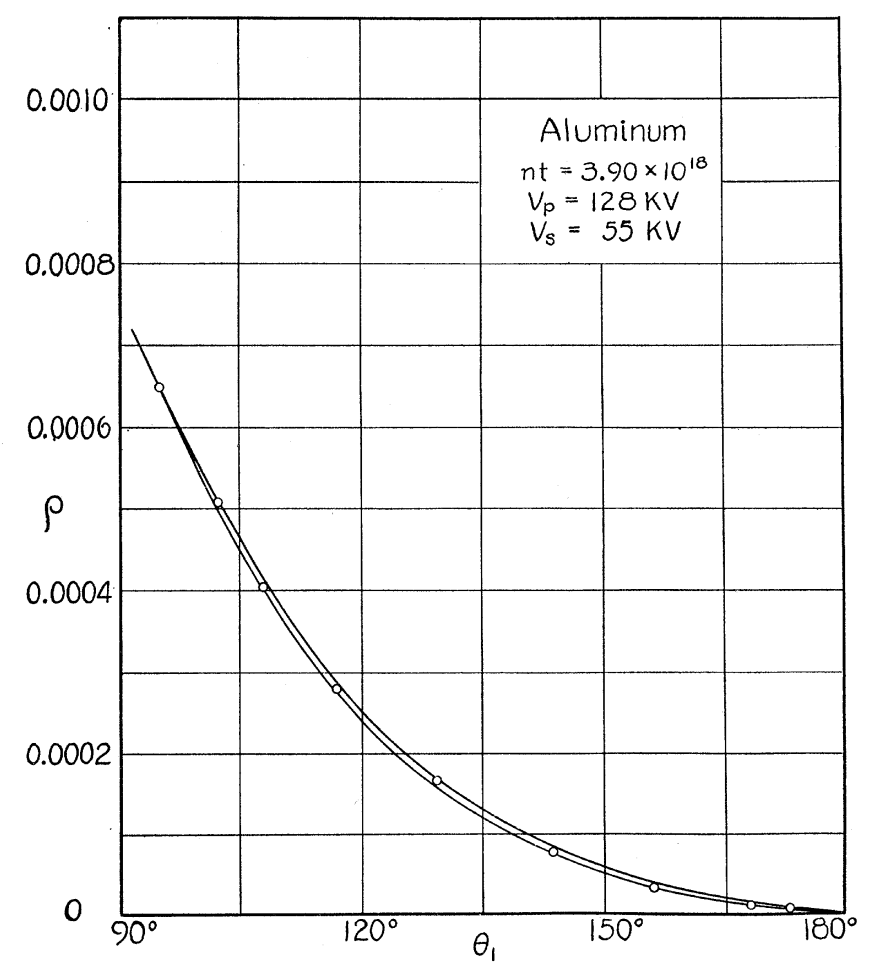

Fig. 11. Lowest curve of Fig. 10 compared with the angular distribution given by Mott and Rutherford. Theoretical curves fitted at $95^{\circ}$.

\section{Angular distribution of secondary electrons.}

To find the dependence on angle of the secondary electrons emitted between two energies, we need only to take the difference in ordinates of two curves for two different stopping potentials. Fig. 12 shows the result for aluminum taken from Fig. 10. It was found that the points agree very well with the curve $k^{\prime}\left(\sin ^{2} \theta_{1}-\sin ^{2} \theta_{2}\right)$. Since the second term in the parenthesis is small compared with the first this may be written as $k^{\prime} \sin ^{2} \theta_{1}$. If this is differentiated with respect to $\theta$ and divided by $\sin \theta$ the result is $2 k \cos \theta$, which represents the intensity of the secondary electrons at the angle $\theta$. The following conclusions may then be drawn: The electrons that come from a thin foil when bombarded with high velocity electrons may be divided into two definite and distinct groups:

1. Those that are scattered without an appreciable loss of energy and have an intensity distribution given by either Rurtherford's $\operatorname{cosec}^{4} \theta / 2$ law, or Mott's equation. 
2. Those that come off with low velocities and have an intensity distribution given by $\cos \theta$. It is interesting to note that the cosine distribution is also obtained for the emission of electrons from solid surfaces.

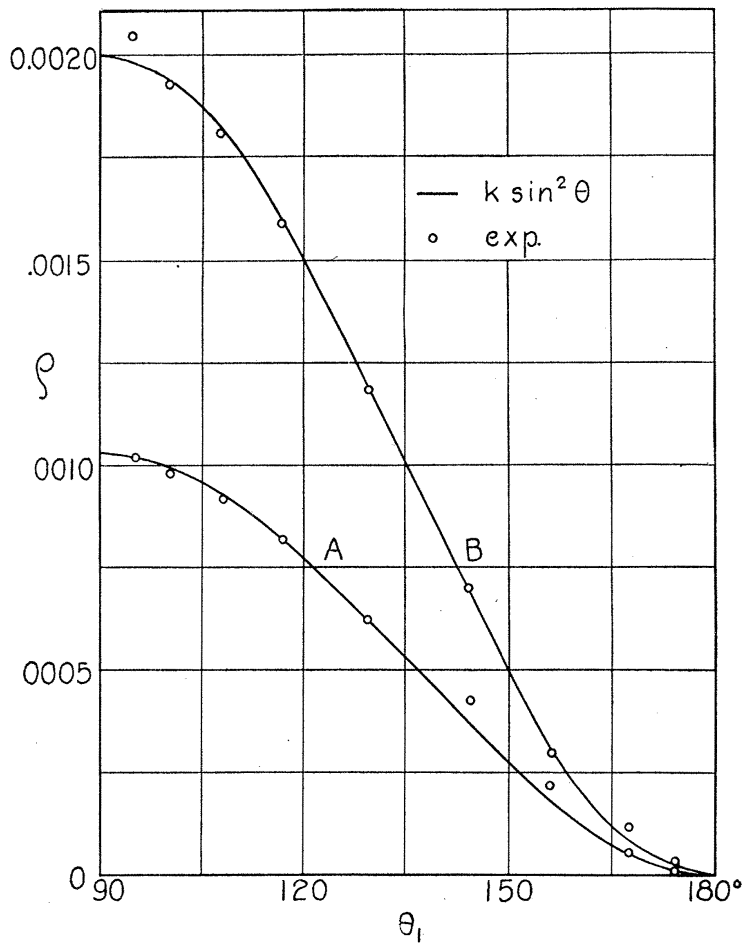

Fig. 12. Angular distribution of secondary electrons from a thin foil taken from Fig. 10. Curve $A, 0$ to $55 \mathrm{kv}$, curve $B, 2$ to $55 \mathrm{kv}$.

\section{Effects Due to Radiation}

When an electron is accelerated energy is lost due to radiation. The continuous $x$-ray spectrum is due to the hyperbolic orbits of the electron around the nuclei of the atoms composing the x-ray target. Some electrons will lose all their energy through radiation and these will give the short wave-length limit or the maximum frequency of the radiation emitted according to the relation of Duane and Hunt, $V e=h \nu_{\max }$. Kramers ${ }^{16}$ has computed the amount of energy lost by an electron deflected through an angle $\theta$ upon the assumption that the orbit is not appreciably disturbed. He finds the expression,

$$
R=\frac{4}{3}\left(\frac{1}{2} m v^{2}\right) \frac{\beta^{3}}{Z} \tan ^{5} \frac{\theta}{2}\left[(\pi+\theta)\left(1+\frac{1}{2} \operatorname{cosec}^{2} \frac{\theta}{2}\right)+3 \cot \frac{\theta}{2}\right] .
$$

For a 50,000 volt electron deflected through $90^{\circ}$ by an aluminum nucleus, the relative amount of energy lost is ten percent according to the above relation.

Mot ${ }^{17}$ has considered the effect of radiation forces on the angular distribu-

${ }^{16}$ H. A. Kramers, Phil. Mag. 46, 826 (1923).

${ }^{17}$ N. F. Mott, Proc. Camb. Phil. Soc. 27, II, 255 (1931). 
tion of nuclearly scattered electrons. Although an exact solution is not obtained, he concludes that the correction to be applied to the scattering equation is an addition of not more than a few percent. However, until a more accurate calculation is made, especially to determine the dependence on angle, no definite conclusions can be drawn.

Qualitative tests may be made experimentally as follows:

1. For large angles it might be expected that many electrons have lost a large portion of their energy and that an appreciable number would be stopped by $-55,000$ volts if the original energy were 110,000 . If this were true the curve showing the variation of $\rho$ with $\theta$ would come close to the axis $\rho=0$ at large angles. No such effect is noticed.

2. Since light atoms 2, according to Kramer's equation cause a greater loss of energy than heavier atoms, we should expect a differently shaped curve connecting $\rho$ and $\theta$ for aluminum and gold. Both elements follow the same law experimentally.

3. The relative loss of energy according to Kramers is proportional to $\beta^{3}$. We should expect, then, that for two widely different potentials withonehalf the primary voltage used as a stopping potential in each case, we should get departures from scattering equations that are based on purely elastic scattering. No such departures are found.

What can be said, then, about the electrons that generate the continuous $\mathrm{x}$-ray spectrum? There seem to be at least two possible explanations. Either, (1) The number losing one-half their energy or more is inappreciable compared with a given fraction of the main beam collected between $140^{\circ}$ and $180^{\circ}$, or, (2) Momentum relations are such as to distribute over various angles those electrons losing energy.

\section{Discussion OF ERRORS}

It is thought that the main error entering into the measurement of $\rho$ experimentally came from an inaccurate knowledge of the "zero correction" when the foil was in place. The foil distributed the electrons going through in a different way than was the case when the "zero correction" was taken with the foil present. It is estimated that this error was not more than 1 or 2 percent; first, because of the small "zero correction" when the foil was absent, (about 0.0002 of the main beam), and second, because with a very thin foil, where the "zero correction" was comparable with the true value of $\rho$, the main beam was not scattered appreciably.

An analysis showed that the error due to the finite size of the beam and its slight divergence was negligible.

To make certain that no appreciable impurities of large atomic number were present in the aluminum foil used, some very pure aluminum from Siegbahn's laboratory was tested. The values of $\rho / n t$ agreed to within 1 percent of those obtained with the foils regularly used.

The value of $n t$ could be determined to 1 percent and the error in measuring the angle on the factor $\cot ^{2} \theta_{1} / 2$ was of the same magnitude.

It may, therefore, be concluded that the experimental error in the absolute 
value of $\rho$ for aluminum cannot be greater than 3 or 4 percent. For gold and silver the error may be slightly larger since only three and not five different foils were used for each in determining the final average values of $\rho / n t$.

\section{Comparison with theory.}

\section{Conclusions}

The experimental results when compared with existing theories may be summarized as follows:

1. Dependence on energy of primary beam: Either Mott's equation or the empirical relation $k / V^{2}$ gives good agreement within the range 56 to $145 \mathrm{kv}$.

2. Dependence on atomic number: According to Mott's equation, $\rho$ increases faster than $Z^{2}$. This is found experimentally but the increase is not sufficient to give good agreement. All other equations give $\rho$ proportional to $Z^{2}$.

3. Dependence on angle: Either Rutherford's or Mott's equation gives good agreement.

4. Absolute values of $\rho$ : Within the ranges $\theta=95^{\circ}$ to $173^{\circ}$, and $V=56$ to $145 \mathrm{kv}$, absolute values of scattering by aluminum are given by, Exp. $=1.32$ Mott.

The classical relativistic result of Darwin as applied by Crowther and Schonland does not agree with the experimental values reported here, either in absolute magnitude or in its dependence on $\theta$ and $\beta$.

The difference in absolute magnitude between theory and experiment is the most outstanding discrepancy. The effect of a nuclear magnetic moment has been computed by Massey ${ }^{18}$ and was found to be negligible. Also, it appears both from an experimental point of view and from Mott's theoretical investigation that radiative forces cannot account for the difference. The explanation must be looked for elsewhere. It might be expected that since the discrepancy becomes less for lighter elements, some information might be obtained by using hydrogen, helium and beryllium. It is planned to extend this work to the case of gases in the near future.

\section{Comparison with other observers.}

The absolute values of $\rho$ for aluminum obtained in this paper are from one-half to two-thirds those obtained by Schonland. The results on silver and gold given here are from 0.7 to 0.8 of his values. It is quite apparent that the difference is mainly due to secondary electrons. A comparison with the work of Chadwick and Mercier can hardly be made since they used much smaller angles and an extrapolation is not permissable at this stage of experimental knowledge.

The writer wishes to take this opportunity to express his appreciation to Professor E. C. Watson who suggested the problem and who has continuously been interested in the work. His sincere thanks are also due Dr. R. A. Millikan for the personal interest he has shown.

${ }^{18}$ H. S. W. Massey, Proc. Roy. Soc. A127, 666 (1930). 